\title{
CERTAIN UNIFIED INTEGRAL FORMULAS INVOLVING THE GENERALIZED MODIFIED K-BESSEL FUNCTION OF FIRST KIND
}

\author{
K. S. NISAR AND S.R. MONDAL
}

\begin{abstract}
Generalized integral formulas involving the generalized modified k-Bessel function $J_{k, \nu}^{c, \gamma, \lambda}(z)$ of first kind are expressed in terms generalized $k$-Wright functions . Some interesting special cases of the main results are also discussed
\end{abstract}

\section{INTRODUCTION}

The integral formula involving various special functions have been studied by many researchers $(7,, 8])$. In 1888 Pincherle gave the integrals involving product of Gamma functions along vertical lines (see [1, 2, 3]). Barnes [4], Mellin [5] and Cahen [6] extended some of these integrals in the study of Riemann zeta function and other Drichlet's series. The integral representation of Fox H-functions and hypergeometric ${ }_{2} F_{1}$ functions studied by [9] and [10] respectively. Also, the integral representation of Bessel functions are given in many recent works (see [11, [12, 7] and 15 ).

let $k \in R ; \alpha, \lambda, \gamma, v \in C ; \operatorname{Re}(\lambda)>0, \operatorname{Re}(v)>0$, the $k$-Bessel function of the first kind defined by the following series [13]:

$$
J_{k, \nu}^{(\gamma),(\lambda)}(z)=\sum_{n=0}^{\infty} \frac{(\gamma)_{n, k}}{\Gamma_{k}(\lambda n+v+1)} \frac{(-1)^{n}(z / 2)^{n}}{(n !)^{2}}
$$

where $(\gamma)_{n, k}$ is the $k$-Pochhammer symbol [25] is defined as:

$$
(x)_{n, k}=x(x+k)(x+2 k) \ldots(x+(n-1) k), \gamma \in C, k \in R \text { and } n \in N
$$

and $\Gamma_{k}(z)$ is the $k$-gamma function, $k$ be the positive real number, defined by (see [25])

$$
\Gamma_{k}(z)=\int_{0}^{\infty} e^{-\frac{t^{k}}{k}} t^{z-1} d t, \operatorname{Re}(z)>0
$$

Clearly, for $k=1, \Gamma_{k}(z)$ reduces to the classical $\Gamma(z)$ function.

In this paper, we introduce a new generalization of $k$-Bessel function called generalized modified $k$-Bessel function and is defined as:

1991 Mathematics Subject Classification. Primary 33B20; 33C20; 26A33; secondary 33B15; $33 \mathrm{C} 05$

Key words and phrases. k-Bessel function; Gamma function; hypergeometric function ${ }_{2} F_{1}$; generalized hypergeometric function ${ }_{p} F_{q}$; generalized (Wright) hypergeometric functions ${ }_{p} \Psi_{q}$; Oberhettinger's integral formula; 
let $k \in R ; \alpha, \lambda_{1}, \gamma, v, c, b \in C ; \operatorname{Re}\left(\lambda_{1}\right)>0, \operatorname{Re}(v)>0$, the generalized modified $k$-Bessel function of the first kind given by the following series

$$
J_{k, \nu}^{c, \gamma, \lambda}(z)=\sum_{n=0}^{\infty} \frac{(c)^{n}(\gamma)_{n, k}}{\Gamma_{k}\left(\lambda_{1} n+v+\frac{b+1}{2}\right)} \frac{(z / 2)^{v+2 n}}{(n !)^{2}}
$$

The aim of this paper is to establish two generalized integral formulas, which are expressed in terms of generalized $k$-Wright functions, by inserting newly generalized modified k-Bessel function.

The generalized Wright hypergeometric function ${ }_{p} \psi_{q}(z)$ is given by the series

$$
{ }_{p} \psi_{q}(z)={ }_{p} \psi_{q}\left[\begin{array}{c}
\left(a_{i}, \alpha_{i}\right)_{1, p} \\
\left(b_{j}, \beta_{j}\right)_{1, q}
\end{array} \mid z\right]=\sum_{k=0}^{\infty} \frac{\prod_{i=1}^{p} \Gamma\left(a_{i}+\alpha_{i} k\right)}{\prod_{j=1}^{q} \Gamma\left(b_{j}+\beta_{j} k\right)} \frac{z^{k}}{k !},
$$

where $a_{i}, b_{j} \in \mathbb{C}$, and real $\alpha_{i}, \beta_{j} \in \mathbb{R}(i=1,2, \ldots, p ; j=1,2, \ldots, q)$. Asymptotic behavior of this function for large values of argument of $z \in \mathbb{C}$ were studied in [17. and under the condition

$$
\sum_{j=1}^{q} \beta_{j}-\sum_{i=1}^{p} \alpha_{i}>-1
$$

was found in the work of [18, 19]. Properties of this generalized Wright function were investigated in 21], (see also [22, 23]. In particular, it was proved 21] that ${ }_{p} \psi_{q}(z), z \in \mathbb{C}$ is an entire function under the condition (1.6).

The generalized hypergeometric function represented as follows [24:

$$
{ }_{p} F_{q}\left[\begin{array}{l}
\left(\alpha_{p}\right) ; z \\
\left(\beta_{q}\right) ; z
\end{array}\right]=\sum_{n=0}^{\infty} \frac{\Pi_{j=1}^{p}\left(\alpha_{j}\right)_{n}}{\Pi_{j=1}^{p}\left(\beta_{j}\right)_{n}} \frac{z^{n}}{n !},
$$

provided $p \leq q ; p=q+1$ and $|z|<1$

where $(\lambda)_{n}$ is well known Pochhammer symbol defined for $($ for $\lambda \in C$ ) (see [24])

$$
\begin{gathered}
(\lambda)_{n}:=\left\{\begin{array}{cc}
1 & (n=0) \\
\lambda(\lambda+1) \ldots .(\lambda+n-1) & (n \in N:=\{1,2,3 \ldots\})
\end{array}\right. \\
(\lambda)_{n}=\frac{\Gamma(\lambda+n)}{\Gamma(\lambda)} \quad\left(\lambda \in C \backslash Z_{0}^{-}\right) .
\end{gathered}
$$

where $Z_{0}^{-}$is the set of non-positive integers.

If we put $\alpha_{1}=\ldots=\alpha_{p}=\beta_{1}=\ldots .=\beta_{q}$ in (1.5), then (1.7) is a special case of the generalized Wright function:

$$
{ }_{p} \psi_{q}(z)={ }_{p} \psi_{q}\left[\begin{array}{l}
\left(\alpha_{1}, 1\right), \ldots,\left(\alpha_{p}, 1\right) ; z \\
\left(\beta_{1}, 1\right), \ldots,\left(\beta_{q}, 1\right) ; z
\end{array}\right]=\frac{\prod_{j=1}^{p} \Gamma\left(\alpha_{j}\right)}{\prod_{j=1}^{q} \Gamma\left(\beta_{j}\right)}{ }_{p} F_{q}\left[\begin{array}{c}
\alpha_{1}, \ldots, \alpha_{p} ; z \\
\beta_{1}, \ldots, \beta_{q} ; z
\end{array}\right]
$$

For the present investigation, we need the following result of Oberbettinger [14]

$$
\int_{0}^{\infty} x^{\mu-1}\left(x+a+\sqrt{x^{2}+2 a x}\right)^{-\lambda} d x=2 \lambda a^{-\lambda}\left(\frac{a}{2}\right)^{\mu} \frac{\Gamma(2 \mu) \Gamma(\lambda-\mu)}{\Gamma(1+\lambda+\mu)}
$$

provided $0<\operatorname{Re}(\mu)<\operatorname{Re}(\lambda)$ 
Also, we need the following relation of $\Gamma_{k}$ with the classical gamma Euler function (see [26]:

$$
\begin{gathered}
\Gamma_{k}(z+k)=z \Gamma_{k}(z) \\
\Gamma_{k}(z)=k^{\frac{z}{k}-1} \Gamma\left(\frac{z}{k}\right) \\
\Gamma_{k}(k)=1
\end{gathered}
$$

\section{Main Results}

Two generalized integral formulas established here, which expressed in terms of generalized $k$-Wright functions (1.10) by inserting the generalized modified $\mathrm{k}$ Bessel function of the first kind (1.4) with the suitable argument in the integrand of (1.11)

Theorem 1. For $\lambda, \mu, \nu, c, \lambda_{1} \in \mathbb{C}, \operatorname{Re}(\lambda+\nu+2)>\operatorname{Re}(\mu)>0$ and $x>0$. Then the following formula holds true:

$$
\begin{aligned}
& \int_{0}^{\infty} x^{\mu-1}\left(x+a+\sqrt{x^{2}+2 a x}\right)^{-\lambda} J_{k, v}^{c, \gamma, \lambda_{1}}\left(\frac{y}{x+a+\sqrt{x^{2}+2 a x}}\right) d x \\
= & 2^{1-\nu-\mu} a^{\mu-\lambda-v} y^{v} k^{-2 \mu} \Gamma(2 \mu) \\
& \times_{k, 2} \Psi_{3}\left[\begin{array}{c}
(\lambda+v+k, 2),(k(\nu+\lambda-\mu), 2 k) ; \\
\left(\nu+\frac{b+1}{2}, \lambda_{1}\right),(k(1+\lambda+v+\mu), 2 k),(\lambda+\nu, 2) ; \frac{c y^{2}}{4 a^{2}}
\end{array}\right]
\end{aligned}
$$

where ${ }_{k, 2} \Psi_{3}$ denote the $k$-Fox-Wright function [26]

Proof. By applying (1.4) to the LHS of (2.1) and interchanging the order of integration and summation, which is verified by uniform convergence of the involved series under the given conditions, we obtain

$$
\begin{aligned}
& \int_{0}^{\infty} x^{\mu-1}\left(x+a+\sqrt{x^{2}+2 a x}\right)^{-\lambda} J_{k, v}^{c, \gamma, \lambda_{1}}\left(\frac{y}{x+a+\sqrt{x^{2}+2 a x}}\right) d x \\
= & \int_{0}^{\infty} x^{\mu-1}\left(x+a+\sqrt{x^{2}+2 a x}\right)^{-\lambda} \\
& \times \sum_{n=0}^{\infty} \frac{(c)^{n}(\gamma)_{n, k}}{\Gamma_{k}\left(\lambda_{1} n+v+\frac{b+1}{2}\right)} \frac{\left(\frac{y}{2}\right)^{v+2 n}}{(n !)^{2}}\left(x+a+\sqrt{x^{2}+2 a x}\right)^{-(v+2 n)} d x \\
= & \sum_{n=0}^{\infty} \frac{(c)^{n}(\gamma)_{n, k}}{\Gamma_{k}\left(\lambda_{1} n+v+\frac{b+1}{2}\right)} \frac{\left(\frac{y}{2}\right)^{v+2 n}}{(n !)^{2}} \int_{0}^{\infty} x^{\mu-1}\left(x+a+\sqrt{x^{2}+2 a x}\right)^{-(\lambda+\nu+2 n)} d x
\end{aligned}
$$

In view of the conditions given in Theorem 1 , since $\mathbb{R}(\lambda+\nu)>\mathbb{R}(\mu)>0$ $k \in N_{0}:=N \cup\{0\}$.

Applying (1.11) to the integrand of (2.1) and obtain the following expression:

$$
\begin{aligned}
= & \sum_{n=0}^{\infty} \frac{(c)^{n}(\gamma)_{n, k}}{\Gamma_{k}\left(\lambda_{1} n+v+1\right)} \frac{\left(\frac{y}{2}\right)^{v+2 n}}{(n !)^{2}} 2(\lambda+\nu+2 n) a^{-(\lambda+\nu+2 n)}\left(\frac{a}{2}\right)^{\mu} \\
& \times \frac{\Gamma(2 \mu) \Gamma(\lambda+\nu+2 n-\mu)}{\Gamma(1+\lambda+\nu+\mu+2 n)}
\end{aligned}
$$


By making the use of the relation (1.12), we obtain

$$
\begin{aligned}
= & 2^{1-\nu-\mu} a^{\mu-\lambda-v} y^{v} k^{-2 \mu} \Gamma(2 \mu) \\
& \times \sum_{k-0}^{\infty} \frac{\Gamma_{k}(\lambda+\nu+k+2 n) \Gamma_{k}(\lambda k+\nu k-\mu k+2 k n)}{\Gamma_{k}(\lambda+\nu 2 n) \Gamma_{k}\left(\lambda_{1} n+v+\frac{b+1}{2}\right) \Gamma_{k}(k+\lambda k+\nu k+\mu k+2 k n)} \\
& \times \frac{(c)^{n}}{(n !)^{2}}\left(\frac{y^{2}}{4 a^{2}}\right)^{n}
\end{aligned}
$$

which is the desired result.

Corollary 1. Let the conditions of Theorem 1 be satisfied and let $k=\lambda_{1}=1$ and $c=-c$ in (2.1). Then the following integral formula holds:

$$
\begin{aligned}
& \int_{0}^{\infty} x^{\mu-1}\left(x+a+\sqrt{x^{2}+2 a x}\right)^{-\lambda} J_{k, v}^{c, \gamma, \lambda_{1}}\left(\frac{y}{x+a+\sqrt{x^{2}+2 a x}}\right) d x \\
= & 2^{1-\nu-\mu} a^{\mu-\lambda-v} y^{v} \Gamma(2 \mu) \\
& \times_{2} \Psi_{3}\left[\begin{array}{c}
(1+\lambda+v, 2),(\nu+\lambda-\mu, 2) ; \\
\left.\left(\nu+\frac{b+1}{2}, \lambda_{1}\right),(1+\lambda+v+\mu, 2),(\lambda+\nu, 2) ; \frac{-c y^{2}}{4 a^{2}}\right]
\end{array}\right.
\end{aligned}
$$

which is the result given by 12 .

Corollary 2. Setting $b=c=1$ in (2.1) with some appropriate parameter replacements, we get the integral formula of Bessel function $J_{v}(z)$ given by Choi and Agarwal [1].

Theorem 2. For $\lambda_{1}, \mu, \nu, c, b \in \mathbb{C}, 0<\mathbb{R}(\mu+\nu+2)<\mathbb{R}(\lambda+\nu+2)$ and $x>0$, then the following integral formula holds true:

$$
\begin{aligned}
& \int_{0}^{\infty} x^{\mu-1}\left(x+a+\sqrt{x^{2}+2 a x}\right)^{-\lambda} J_{k, v}^{c, \gamma, \lambda_{1}}\left(\frac{x y}{x+a+\sqrt{x^{2}+2 a x}}\right) d x \\
= & 2^{1-2 \nu-\mu} y^{\nu} a^{\mu-\lambda} k^{1+\lambda-\mu} \Gamma(\lambda-\mu) \\
& \times_{k, 2} \Psi_{3}\left[\begin{array}{c}
(k(2 \mu+2 \nu), 4 k),(\nu+\lambda+k, 2) ; \\
\left(\nu+1, \lambda_{1}\right),(\nu+\lambda, 2),(k(1+\lambda+\mu+2 \nu), 4 k) ;
\end{array} \quad \frac{c y^{2}}{4}\right]
\end{aligned}
$$

where ${ }_{k, 2} \Psi_{3}$ denote the $k$-Fox-Wright function [26]

Proof. By applying (1.4) to the LHS of (2.2) and interchanging the order of integration and summation, which is verified by uniform convergence of the involved series under the given conditions, we obtain 


$$
\begin{aligned}
& \int_{0}^{\infty} x^{\mu-1}\left(x+a+\sqrt{x^{2}+2 a x}\right)^{-\lambda} J_{k, v}^{c, \gamma, \lambda_{1}}\left(\frac{x y}{x+a+\sqrt{x^{2}+2 a x}}\right) d x \\
= & \int_{0}^{\infty} x^{\mu-1}\left(x+a+\sqrt{x^{2}+2 a x}\right)^{-\lambda} \\
& \times \sum_{n=0}^{\infty} \frac{(c)^{n}(\gamma)_{n, k}}{\Gamma_{k}\left(\lambda_{1} n+v+\frac{b+1}{2}\right)} \frac{\left(\frac{x y}{2}\right)^{v+2 n}}{(n !)^{2}}\left(x+a+\sqrt{x^{2}+2 a x}\right)^{-(v+2 n)} d x \\
= & \sum_{n=0}^{\infty} \frac{(c)^{n}(\gamma)_{n, k}}{\Gamma_{k}\left(\lambda_{1} n+v+\frac{b+1}{2}\right)} \frac{\left(\frac{y}{2}\right)^{v+2 n}}{(n !)^{2}} \\
& \times \int_{0}^{\infty} x^{(\mu+v+2 n)-1}\left(x+a+\sqrt{x^{2}+2 a x}\right)^{-(\lambda+v+2 n)} d x
\end{aligned}
$$

Applying (1.11) to the integrand of (2.2) ,we obtain the following expression:

$$
\begin{aligned}
= & \sum_{n=0}^{\infty} \frac{(c)^{n}(\gamma)_{n, k}}{\Gamma_{k}\left(\lambda_{1} n+v+\frac{b+1}{2}\right)} \frac{\left(\frac{y}{2}\right)^{v+2 n}}{(n !)^{2}} 2(\lambda+v+2 n) a^{-(\lambda+v+2 n)}\left(\frac{a}{2}\right)^{\mu+v+2 n} \\
& \times \frac{\Gamma(2 \mu+2 \nu+4 n) \Gamma(\lambda-\mu)}{\Gamma(1+\lambda+2 \nu+\mu+4 n)}
\end{aligned}
$$

By making the use of (1.12), we obtain

$$
\begin{aligned}
= & 2^{1-2 \nu-\mu} y^{\nu} a^{\mu-\lambda} k^{\lambda-\mu+1} \Gamma(\lambda-\mu) \\
& \times \sum_{k-0}^{\infty} \frac{(\gamma)_{n, k} \Gamma_{k}(\lambda+\nu+k+2 n) \Gamma_{k}(2 \mu k+2 \nu k+4 n k)}{(n !)^{2} \Gamma_{k}\left(\lambda_{1} n+v+\frac{b+1}{2}\right) \Gamma_{k}(\lambda+\nu+2 n) \Gamma_{k}((1+\lambda+\mu+2 v+4 n) k)}\left(\frac{c y^{2}}{4}\right)^{n}
\end{aligned}
$$

which is the desired result.

Corollary 3. Let the conditions given in Theorem 2 satisfied and set $k=\lambda_{1}=$ 1 , and $c=-c$ Theorem 2 reduces to

$$
\begin{aligned}
& \int_{0}^{\infty} x^{\mu-1}\left(x+a+\sqrt{x^{2}+2 a x}\right)^{-\lambda} J_{1, v}^{c, \gamma, 1}\left(\frac{x y}{x+a+\sqrt{x^{2}+2 a x}}\right) d x \\
= & 2^{1-2 \nu-\mu} y^{\nu} a^{\mu-\lambda} \Gamma(\lambda-\mu) \\
& \times_{2} \Psi_{3}\left[\left(\nu+\frac{b+1}{2}, \lambda_{1}\right),(\nu+\lambda, 2),(1+\lambda+\mu+2 \nu, 4) ; \frac{c y^{2}}{4}\right]
\end{aligned}
$$

which is the result given by [12] .

Corollary 4. setting $b=c=1$ in (2.1) with some appropriate parameter replacements, we get the integral formula of Bessel function $J_{v}(z)$ given by Choi and Agarwal [1].

\section{Conclusion}

The integral formulas for generalized modified $k$-Bessel function of first kind is derived and the results are expressed interm of generalized $k$-Wright function. Some of interesting special cases also derived from the main results. Using some suitable parametric replacement, theorems 1 and 2 gives the unified integral representation of generalized Bessel function, if $c=-1$ and integral representation of modified Bessel function by, if $c=1$. 


\section{REFERENCES}

[1] S. Pincherle, Sulle funzioni ipergeometriche generalizzate Nota I, Atti della Reale Accademia dei Lincei. Rendiconti della Classe di Scienze Fisiche, Matematiche e Naturali, Serie 4, Vol. 4 (1888), 694-700

[2] Pincherle, S: Sulle funzioni ipergeometriche generalizzate. Nota I-II. In: Opere Scelte-I, Unione Matematica Italiana, Ed. Cremonese, Rome (1954), 223-239.

[3] Mainardi, F, Pagnini, G, Pincherle, S: The pioneer of the Mellin-Barnes integrals. J. Comput. Appl. Math. 153, (2003),331-342.

[4] Barnes, EW: A new development of the theory of the hypergeometric functions. Proc. Lond. Math. Soc. 6, 141-177, (1908)

[5] Mellin, HM: Abriß einer einheitlichen theorie der Gamma und der hypergeometrischen funktionen. Math. Ann. 68, 305-337 (1910)

[6] Cahen, E: Sur la fonction $\xi \sigma$ de Riemann et sur des fonctions analogues. Ann. Sci. Éc. Norm. Super. 11, 75-164, (1894)

[7] Brychkov, YA: Handbook of special functions: derivatives, integrals, series and other formulas. CRC Press, Boca Raton, (2008)

[8] Choi, J, Hasanov, A, Srivastava, HM, Turaev, M: Integral representations for Srivastava's triple hypergeometric functions. Taiwan. J. Math. 15, 2751-2762 (2011)

[9] Garg, M, Mittal, S: On a new unified integral. Proc. Indian Acad. Sci. Math. Sci. 114(2), 99-101 (2003)

[10] Ali, S: On some new unified integrals. Adv. Comput. Math. Appl. 1(3), 151-153 (2012)

[11] Junesang Choi, Praveen Agarwal, Certain unified integrals associated with Bessel functions, Boundary Value Problems 2013, 2013:95

[12] J.Choi, P.Agarwal,S.Mathur and S.D.Purohit, Certain new integral formulas involving the generalized Bessel functions,Bull. Korean Math. Soc., 4(51),2014, 995-1003.

[13] L.G.Romero, G.A.Dorrego and R.A. Cerutti, The k-Bessel function of first kind,International Mathematical forum,38(7), 1859-1854.

[14] Oberhettinger, F: Tables of Mellin Transforms. Springer, New York (1974)

[15] Watson, GN: A Treatise on the Theory of Bessel Functions. Cambridge Mathematical Library Edition. Camdridge University Press, Camdridge (1995). Reprinted (1996)

[16] A. Erdélyi, W. Magnus, F. Oberhettinger and F.G. Tricomi, Higher transcendental functions. Vols. I, II, McGraw-Hill Book Company, Inc., New York, 1953. NewYork, Toronto, London, 1953.

[17] C. Fox, The asymptotic expansion of generalized hypergeometric functions, Proc. London. Math. Soc. 27 (1928), no. 4, 389-400.

[18] E. M. Wright, The asymptotic expansion of integral functions defined by Taylor series, Philos. Trans. Roy. Soc. London, Ser. A. 238 (1940), 423-451.

[19] E. M. Wright, The asymptotic expansion of the generalized hypergeometric function, Proc. London Math. Soc. (2) 46 (1940), 389-408.

[20] A. A. Kilbas and M. Saigo,H-transforms, Chapman \& Hall/CRC, Boca Raton, FL, 2004.

[21] A. A. Kilbas, M. Saigo and J. J. Trujillo, On the generalized Wright function, Fract. Calc. Appl. Anal. 5 (2002), no. 4, 437-460.

[22] A. A. Kilbas and N. Sebastian, Generalized fractional integration of Bessel function of the first kind, Integral Transforms Spec. Funct. 19 (2008), no. 11-12, 869-883.

[23] A. A. Kilbas and N. Sebastian, Fractional integration of the product of Bessel function of the first kind, Fract. Calc. Appl. Anal. 13 (2010), no. 2, 159-175.

[24] E. D. Rainville, Special functions, Macmillan, New York, 1960.

[25] R. Diaz and E. Pariguan, On hypergeometric functions and $k$-Pochhammer symbol, Divulgaciones Mathematicas, Vol. 15, 2(2007), pp 179-192.

[26] R. A. Cerutti, On the $k$-Bessel functions, International Mathematical forum, Vol. 7, 38(2012), pp. 1851-1857. 
Department of Mathematics, College of Arts \& Science-Wadi Addwaser, Prince Sattam bin Abdulaziz University, Saudi Arabia

E-mail address: ksnisar1@gmail.com

Department of Mathematics, College of Science-Al Ahsa, King Faisal University, SAUdi ARABia

E-mail address: saiful786@gmail.com 